\title{
APLICAÇÃO DE REDES DE SENSORES SEM FIO NA AGRICULTURA DE PRECISÃO: UMA REFLEXÃO TEÓRICA
}

Melissa Bonfim Alcantud, Paulo Roberto Rosa

Instituto Federal de Educação, Ciência e Tecnologia de São Paulo - IFSP, Curso Superior de Tecnologia em Análise e Desenvolvimento de Sistemas, Campus de Presidente Epitácio, SP. E-mail: melissa.alcantud@gmail.com

\section{RESUMO}

Atualmente o uso de tecnologias sem fio tem proporcionado o avanço nas mais diversas áreas, como é o caso da agricultura de precisão. O uso de Rede de Sensores Sem Fios em culturas agrícolas permite realizar o monitoramento e controle das condições ambientais. A comunicação entre os sensores tem como finalidade executar as tarefas de sensoriamento, coleta, monitoramento, transmissão e processamento de informações. O objetivo deste artigo é analisar a aplicação das Redes de Sensores Sem Fios na agricultura de precisão, bem como sua viabilidade, formas de utilização e problemas relacionados. Todo o estudo desenvolvido neste artigo encontrase embasado pesquisas bibliográficas compostas por livros e artigos eletrônicos de renomados autores.

Palavras-chave: redes sem fio, sensoriamento, agricultura de precisão, tecnologia, energia.

\section{APPLICATION OF WIRELESS SENSOR NETWORKS IN PRECISION AGRICULTURE: A THEORETICAL REFLECTION}

\begin{abstract}
Currently the use of wireless technologies has provided the advance in the most diverse areas, as it is the case of the precision agriculture. The use of Wireless Sensor Network in agricultural crops allows the monitoring and control of environmental conditions. The communication between the sensors has as purpose to perform the tasks of sensing, collecting, monitoring, transmitting and processing information. The objective of this article is to analyse the application of Wireless Sensor Networks in precision agriculture, as well as its viability, usage forms and related problems. All the study developed in this article is based on bibliographic research composed by books and electronic articles of renowned authors.
\end{abstract}

Keywords: wireless networks, sensing, precision agriculture, technology, energy. 


\section{INTRODUÇÃO}

Com o constante avanço tecnológico experimentado a partir da segunda metade do século $X X$, a procura por tecnologias de conexão sem fio vem crescendo cada vez mais e demandando um maior volume de investimentos, de um lado para pesquisa e desenvolvimento de novos produtos e, de outro, para aquisição dos dispositivos necessários à aplicação prática. As Redes de Sensores Sem Fio (RSSF) possuem uma gama de possibilidades de serem utilizadas em diferentes áreas, tais como agricultura, militar, ambiental, médica, entre outras.

A aplicação das redes de sensores sem fio no acompanhamento de culturas agrícolas pode contribuir nas tomadas de decisões, no aumento da produtividade e no uso racional da água. As principais vantagens na utilização das Redes de Sensores Sem Fio (RSSFs) são a ausência de cabeamento e a flexibilidade, podendo ser utilizada principalmente em áreas de difícil acesso, com maior agilidade na instalação - na modificação, quando for o caso -, menor custo operacional, podendo, inclusive, conciliar diferentes topologias. Em contrapartida, a restrição de energia consumida pelos nós sensores deve ser considerada, uma vez que são alimentados por baterias. $O$ objetivo deste texto consiste em analisar a aplicação das redes de sensores sem fio na agricultura de precisão, bem como sua viabilidade, formas de utilização e problemas relacionados.

\section{METODOLOGIA}

Todo o estudo desenvolvido e materializado neste artigo encontra-se embasado em análises provenientes de referencial bibliográfico composto por livros e artigos eletrônicos de renomados autores. Vergara (2000) salienta que a principal vantagem da pesquisa bibliográfica reside ao fato de fornecer uma visão analítica para a temática abordada. Para Lakatos e Marconi (2001, p. 183), “[...] toda bibliografia já tornada pública em relação ao tema estudado [...]" objetiva "[...] colocar o pesquisador em contato direto com tudo o que foi escrito, dito ou filmado sobre determinado assunto".

\section{AGRICULTURA DE PRECISÃO}

No Brasil, a agricultura é uma das atividades econômicas mais importantes. Independente do tipo e do tamanho da exploração agrícola, no conjunto trata-se de uma atividade altamente dependente e influenciada pelas condições climáticas. Especificamente no caso da agricultura de precisão (AP), aquela normalmente praticada em grandes extensões de terra e que utiliza intensivamente os recursos de tecnologia da informação para prover dados geográficos referentes ao solo e clima, o desenvolvimento técnico e tecnológico também apresenta-se como elemento de grande importância. As mudanças nesses elementos - clima, técnica e tecnologia - podem provocar grandes impactos econômicos, sociais e ambientais.

A agricultura de precisão (AP) tem como objetivo o gerenciamento dos sistemas de produção agrícola de modo macro, isto é, desde a aplicação de insumos até a utilização de mapeamentos em todos os processos envolvidos na produção (EMPRAPA, 2011). É por essa razão que a agricultura de precisão vem se utilizando de novas soluções de instrumentação agrícola (OLIVEIRA, 2009).

Por demandar um volume de investimentos considerável, a agricultura de precisão é normalmente empregada em grandes extensões de terra, o que torna a relação custo-benefício mais favorável. Segundo Oliveira (2009), muitos agricultores são refratários a realização de investimentos que envolvam novas tecnologias e quando o retorno do capital investido ocorra no longo prazo. Por essa razão, a realização de um planejamento consistente apresenta-se como um instrumento fundamental para demonstrar os benefícios a serem proporcionados pela adoção de determinada tecnologia. 


\section{REDES SEM FIO}

A tecnologia sem fio já é uma realidade para maioria das pessoas, trazendo uma série de novas funcionalidades para troca de informações sem fio. A mobilidade e a visualização rápida de informações, independentemente da localização, procuram atender a necessidade de seus usuários, de forma simples e rápida. Segundo Soares, Lemos e Colcher (1995):

Nas redes sem fio (Wirelless networks) os pacotes são transmitidos "através do ar", em canais de frequência de rádio (radiodifusão) (frequências na faixa de $\mathrm{KHZ}$ até $\mathrm{GHZ}$ ) ou infravermelho (frequência de $\mathrm{THz}$ ). As redes sem fio (baseadas em radiodifusão) são uma alternativa viável onde é difícil, ou mesmo impossível, instalar cabos metálicos ou de fibra ótica. Seu emprego é particularmente importante para comunicações entre computadores portáteis em um ambiente de rede local móvel (SOARES; LEMOS; COLCHER, 1995, p. 102-103).

As redes sem fio possuem parte de sua estrutura invisível, onde os dispositivos móveis possuem contato direto com uma central de comunicação, conhecida como estação base ou Ponto de Acesso (Access Point - AP) (Kurose; Ross, 2006). Nesse sentido, para Saccol e Reinhard (2007), as redes sem fio são:

[...] tecnologias de informação que envolvem o uso de dispositivos conectados a uma rede ou a outro aparelho por links de comunicação sem fio, como, por exemplo, as redes de telefonia celular ou a transmissão de dados via satélite, além das seguintes tecnologias: Infra-vermelho (infrared - IR), Bluetooth, Wireless LAN (Rede Local sem fio) (SACCOL; REINHARD, 2007, p. 5).

As redes de sensores sem fio (RSSFs) apresentam como principal diferença a inserção de sensores nas redes sem fio. Na agricultura de precisão esses sensores podem ser de multimídia (como captura de imagem em movimento) ou escalares (como temperatura, pressão, umidade do solo e nível de radiação solar).

\section{REDES DE SENSORES SEM FIO}

As RSSFs (WSNs - Wireless Sensor Networks) são utilizadas para a coleta, processamento e disseminação de dados por meio de comunicação sem fio, sobre fenômenos ocorridos em uma região de interesse. Essas redes são compostas por vários dispositivos de hardware, conhecidos como nós sensores, compostos por microcontrolador, bateria, unidade de comunicação de dados e sensores (temperatura, umidade do ar e solo, dentre outros). Desse modo, cada nó sensor monitora seu ambiente local e repassa os dados coletados e processados a um nó central, chamado de nó sink, cuja função é agregar todas as informações recebidas e enviá-las a um sistema controlador, comumente conhecido como Ponto de Acesso (PA), o que não acontece com as redes ad hoc sem fios (Mobile Ad hoc Networks -MANET). Segundo Tanenbaum e Wetherall (2011), as redes de sensores sem fio (RSSFs) são:

[...] implantadas para monitorar aspectos do mundo físico. Os nós sensores são pequenos computadores, normalmente do tamanho de um token de segurança, que possuem sensores de temperatura, vibração e outros. Muitos nós são colocados no ambiente a ser monitorado. Normalmente, eles possuem baterias, embora possam recolher energia de vibrações ou do sol. Assim como a RFID, ter energia suficiente é um desafio importante, e os nós precisam se comunicar cuidadosamente para poderem entregar suas informações de sensoriamento a um ponto de coleta externo. Uma estratégia comum é que os nós se auto -organizem para repassar 
mensagens uns para os outros [...]. Esse projeto é chamado rede de múltiplo saltos (ou rede multihop) (TANENBAUM; WETHERALL, 2011, p. 46 , grifos dos autores).

Para Tavares (2009), do ponto de vista de organização, as RSSFs e as MANETs são iguais, já que os nós se comunicam diretamente entre si através de sequências de saltos com comunicação sem fios. Mas as RSSFs possuem algumas características particulares, tais como a utilização de recursos restritos de energia, uma topologia de rede dinâmica e uma grande quantidade de nós.

Vale salientar que, para Verona (2010), as RSSFs possuem diferentes tipos de configurações, de forma que cada uma seja planejada com suas peculiaridades, tornando cada aplicação de RSSF inviável para outros projetos. Ruiz (2003), qualifica as configurações quanto a:

- Composição: podendo ser homogênea ou heterogênea, conforme a composição da capacidade do hardware dos nós sensores.

- Mobilidade: podendo ser estacionária, quando todos os dispositivos permanecem no mesmo local onde foram depositados durante todo o tempo de vida da rede; ou móvel, quando os dispositivos são deslocados do local onde inicialmente foram depositados.

- Densidade: podendo ser balanceada, conforme a rede apresente a concentração e distribuição de dispositivos por unidade de área. Pode ainda ser a rede ser densa (alta) ou esparsa (baixa), de acordo com concentração de dispositivos por unidade de área.

- Distribuição: a rede pode ser regular, quando a rede apresenta a distribuição uniforme de nós sobre a área monitorada; ou irregular, quando a rede apresenta uma distribuição não uniforme de nós sobre a área monitorada.

- Tamanho: sendo classificada em pequena, a rede composta de uma centena de elementos de rede; média, composta de centenas a mil elementos de rede; ou grande, quando a rede é composta por milhares de elementos de rede.

Também é possível classificar as redes de sensores sem fio de acordo com os tipos empregados nas configurações, de acordo com o tipo de disseminação de dados, conexão, transmissão e fluxo de informação. Outra característica refere-se ao modo de sensoriamento, ou seja, a forma de coletar os dados emitidos pelos nós sensores através do sensoriamento do ambiente analisado. De acordo com Ruiz (2003), são três modos diferentes de coleta:

- Coleta periódica - os dados são coletados em intervalos regulares de tempo.

- Coleta contínua - os dispositivos da rede coletam dado de forma contínua.

- Coleta reativa - a coleta dos dados dado ocorre quando algum evento acontecer ou quando o observador solicitar o recebimento de dados.

Como o modo de coleta está diretamente ligado ao consumo de energia, que por sua vez apresenta-se como um gargalo às RSSFs, a escolha do modo ideal terá impacto direto no planejamento e implementação da infraestrutura necessária. É importante salientar que o modo de coleta contínuo apresenta um maior consumo de energia, o que por sua vez compromete a autonomia da rede.

\section{CONSUMO DE ENERGIA}

A baixa capacidade de potência dos nós sensores, associado ao menor alcance da comunicação entre eles, são quesitos relevantes na implantação de uma RSSF. Diante disso, Tavares (2009) explica que:

O processamento central, ao nível do nó sensor, é realizado por um processador cujo consumo depende da frequência do relógio. Tipicamente este consumo de energia é proporcional à frequência do relógio. Para 
prolongar a vida útil das baterias é possível carregá-las utilizando alguma energia do meio envolvente. Como as RSSFs devem executar tarefas colaborativas, onde é importante detectar e estimar o aparecimento de eventos (e não apenas fornece mecanismos de comunicação), normalmente, os dados são resumidos ou tratados (em clusters) para melhorar o desempenho no processo de detecção de acontecimentos (TAVARES, 2009, p. 24).

Como o consumo de energia vai depender da configuração dos nós sensores, a escolha desses dispositivos será responsável por reduzir ou aumentar significativamente o custo final do projeto. No ramo agrícola, por exemplo, uma coleta de dados pode significar maior capacidade do produtor rural controlar a produtividade de sua lavoura. Porém, para evitar desperdícios, faz-se necessário avaliar e especificar o volume adequado das coletas. Como ferramenta que pode contribuir na concepção de uma rede que atenda as reais necessidades e sem incorrer em desperdícios de recursos temos os simuladores. Através das ferramentas de simulações é possível obter resultados do total de energia que será gasto em cada nó sensor ou na rede inteira.

\section{CONSIDERAÇÕES FINAIS}

As análises realizadas neste artigo apontam para um cenário promissor quanto a aplicação de redes de sensores sem fio na agricultura de precisão. A aplicação das RSSFs na agricultura tem como principal objetivo monitorar variáveis que possibilitem o aprimoramento da atividade, melhorando a produtividade e reduzindo custos operacionais. Cada empreendimento demandará uma RSSF que considere suas especificidades e necessidades, o que implica na concepção de redes específicas para cada projeto.

O uso consolidado de diversas soluções através de RSSFs possibilitam que o empreendimento agrícola tome decisões relacionadas ao trato cultural mediante a destinação de recursos de tipo e em quantidade adequadas às reais necessidades (sementes, fertilizantes, pesticidas, água, etc). Dessa forma busca-se propiciar as condições para aumentar a produtividade, reduzir custos e produzir o menor impacto, tornando o empreendimento mais sustentável. Considera-se, dessa forma, que as RSSFs referem-se a aplicação prática de uma tecnologia que possibilitará avanços produtivos à agricultura.

\section{REFERÊNCIAS}

EMBRAPA Instrumentação. Agricultura de precisão: um novo olhar. São Paulo: Cubo, 2011.

GOMES, R. D., et al. Modelo para simulação realista de redes de sensores sem fio industriais. XXXIII Simpósio Brasileiro de Telecomunicações. Minas Gerais. Set. 2015. Disponível em: <https://www.researchgate.net/publication/281559505_Modelo_para_Simulacao_Realista_de_R edes_de_Sensores_sem_Fio_Industriais>. Acesso em: 03. set. 2016.

KUROSE, J. F.; Ross, K. W. Redes de computadores e a internet: uma abordagem topdown. 3. ed. São Paulo: Pearson, 2006.

LAKATOS, E. M.; MARCONI, M. A. Fundamentos de metodologia científica. 4. ed. São Paulo: Atlas, 2001.

OLIVEIRA, R. P. Agricultura de precisão: a tecnologia da informação em suporte ao conhecimento agronômico clássico. Revista Tecnológica e Cultura, n. 15, 2009, p. 63-71. 
Ruiz, L. B. MANNA: uma arquitetura para o gerenciamento de redes de sensores sem fio. Tese de doutorado - Universidade Federal de Minas Gerais, 2003.

SACCOL, M. Z. REINHARD, N. Tecnologias de informação móveis, sem fio e ubíquas: definições, estado-da-arte e oportunidades de pesquisa. Revista de Administração Contemporânea,vol. 11, n. 4. Curitiba, 2007. <http://www.scielo.br/scielo.php?pid=S141565552007000400009\&script=sci_arttext>Acesso em: 27. nov. 2016.

SOARES, L. F; G.; LEMOS, G.; COLCHER, S. Redes de computadores: das LANs MANs e WANs as redes ATM. 2. ed. Rio de Janeiro: Campus, 1995.

TANenbaum, A. S, WetherALL, D. Redes de computadores. 5. ed. São Paulo: Pearson, 2011.

TAVARES, J. M. S. Desenvolvimento, simulação e validação de protocolos MAC e de encaminhamento para redes de sensores sem fios. Dissertação de Mestrado em Engenharia Eletromecânica - Universidade da Beira Interior. Covilhã, Agosto de 2009.

VERGARA, S. C. Projetos e relatórios de pesquisa em administração. 3. ed. Rio de Janeiro: Atlas, 2000.

VERONA, A. B. Simulação e análise de redes de sensores sem fio aplicadas a viticultura. 2010. $93 \mathrm{f}$. Dissertação de Mestrado em Ciência da Computação - Universidade Estadual de Maringá. 